\title{
A progression strategy of proximal algorithm for the unconstrained optimization
}

\author{
Marouane Nazih \\ LRIT, Associated Unit to CNRST (URAC 29) \\ IT Rabat Center, \\ Faculty of Sciences In Rabat, \\ MOHAMMED V UNIVERSITY IN RABAT, \\ B.P.1014 RP, Rabat, Morocco \\ Email: marouane.nazih1@gmail.com
}

\author{
Khalid Minaoui \\ LRIT, Associated Unit to CNRST (URAC 29) \\ IT Rabat Center, \\ Faculty of Sciences In Rabat, \\ MOHAMMED V UNIVERSITY IN RABAT, \\ B.P.1014 RP, Rabat, Morocco \\ Email: khalid.minaoui@fsr.ac.ma
}

\begin{abstract}
In order to accurately solve the unconstrained optimization problem in which the objective function is nonlinear, a new optimization method different from both linear search and trust region is presented in this paper. This new method is based on the family of proximal algorithms. We customize our algorithm to solve different unconstrained optimization problems, and we verify the theoretical of the proposed method via different numerical examples where we compare the new algorithm with some existing state-of-the-art algorithms. Finally the simulation results proved the performance of the algorithm and indicates the advantage of the proposed algorithm in the case where the hypotheses are verified.
\end{abstract}

Keywords-Optimization, Unconstrained optimization problems, Proximal algorithm.

\section{INTRODUCTION}

Optimization refers to finding an argument that minimizes or maximizes a given function (quadratic function, nonlinear function, the sum of squares function, etc.). Many optimization algorithms seek to find the solution by solving either linear or nonlinear equations by defining the derivative of the objective function equal to zero [1].

Unconstrained optimization problems consider the problem of minimizing an objective function that depends on real variables with no restrictions on their values.

Mathematically, let $\mathrm{x} \in R^{N}$ be a real vector with $\mathrm{N} \geq 1$ components and let f : $R^{N} \mapsto \mathrm{R}$ be a nonlinear smooth function, then our unconstrained optimization problem could be formulated as follows:

$$
\min _{x} f(x)
$$

Unconstrained optimization problems arise directly in several applications in Numerical Optimization [2] and in Cloud Computing [3] [4], but they also arise indirectly from reformulations of constrained optimization problems, that has a wide range of applications in Energy efficient communications [5] [6], and also in Internet of Things (IoT) [7] to recover in a stable way a matrix from the matrix of detection data, note that constrained problems can often be transformed into unconstrained problems with the help of Lagrange multipliers [8], and it is practical to replace the constraints of an optimization problem with penalized terms in the objective function, then solve the problem as an unconstrained problem.

In many nonlinear optimization problems, the objective function has a large number of local minima (and maxima). Finding an arbitrary local optimum is relatively straightforward by using classical local optimization methods, but finding the global minimum (or maximum) of a function is far more difficult where the analytical methods are frequently not applicable, and the use of numerical solution strategies often leads to very hard challenge. In our case, where the objective function is nonlinear, many optimization methods use strategies to ensure that some subsequence of iterations converges to an optimal solution. There are two main families of global strategies for solving problem (1), the first and the most popular for ensuring convergence relies on line search and the second and increasingly popular is trust region.

At every iteration $\mathrm{k}$, the following iterate $x^{(k+1)}$ in both line search and trust region is construct as:

$$
x^{(k+1)}=x^{(k)}+\rho^{(k)} d^{(k)}
$$

by controlling the direction $d^{(k)}$ and the step length $\rho^{(k)}$ - the distance of the move - in order to force the descent condition:

$$
f\left(x^{(k)}+\rho^{(k)} d^{(k)}\right)<f\left(x^{(k)}\right)
$$

both strategies differ in the order in which they choose the direction and the step length, Line search strategy chooses the direction first, followed by the distance, conversely of the Trust-region strategy that firstly chooses the maximum distance, followed by the direction.

It is well known in the philosophy of optimization that strategies for rapid convergence and robustness of the algorithms is often in direct conflict with the requirements of computer storage and speed, for example the gradient method is simple to compute, but it is quite slow and converges but under often complex conditions of convergence [9]. There is also the newton's method that minimizes a function using knowledge of its second derivative, it can be faster for good 
initial guesses when the second derivative is known and easy to compute. However, the analytic expression for the second derivative is often complicated or intractable, and the computation of the second derivative also requires a lot of computation.

On other hand, the trust region methods are later developed than line search, and the former ones have better convergence properties in general, as they change not only the length of the search vector, but also his direction. However, solving the trust region subproblem at each iteration is more expensive than a simple line search, so the former may not pay off for largescale problems [10]. For large-scale problems, a good strategy is to use the conjugate gradient algorithm which ends in at most $\mathbf{N}$ steps in theory (with $\mathbf{N}$ is the size of the problem), but can take much more than $\mathbf{N}$ steps or even do not converge if the problem is ill-conditioned [9].

In case that the problem is ill-conditioned, an effective and practical way is by introducing a regularization to our objective function, instead of going fancy with these globalization algorithms.

Thus, the motivation of this paper is to develop a robust algorithm for unconstrained optimization problems, which can be faster and more accurate compared to others algorithms. The proposed algorithm belongs to a third family of global strategies called the proximal methods, it introduce a regularization term to our objective function (1) to penalize the distance between two iterations. The problem (1) benefits from the strongly convex property [15], which allows us to reach the global minimum in a small number of iterations, by introducing a simple strategy of progression, proximal algorithm's basic parameter $\mu$ to correctly parameterizes the intensity of the penalty, which is adapted from iteration to another.

The rest of the paper is organized as follows. In Section 2 , we review the different approaches of unconstrained optimization while addressing the main algorithms that belong to each approach. In Section 3 we introduce our new optimization algorithm. In Section 4 we deal with analysis of the simulation results and finally Section 6 concludes the paper.

\section{RELATED WORK}

The principle of iterative methods is to generate an improvement sequence of approximate solutions, in which the $\mathrm{k}$-th approximation is derived from the previous ones. They start at a point $\mathbf{x}^{(0)}$ and generate a sequence $\left\{\mathbf{x}^{(k)}\right\}$ that is interrupted when no progress can be made or when a solution seems to have approached with sufficient accuracy. To decide how to go from one iteration $\mathbf{x}^{(k)}$ to the next one, these methods are based on local information on $f$ obtained at the point $\mathrm{x}^{(k)}$ and possibly at the preceding points. They construct a local approximation $\mathbf{m}^{(k)}(\mathbf{x})$ (Figure 1) - the Taylor Young limited development in the current point $\mathbf{x}^{(k)}$ - that is used to solve a local problem, and find a new iterated $\mathbf{x}^{(k+1)}$ for which the value of the objective function will be lesser. However, these algorithms must regularly return to the main objective function $f(x)$ And can not indefinitely deal with the ersatz that is $\mathbf{m}^{(k)}(\mathbf{x})$, At the risk of being misled dangerously. This step that consists of going back from the local problem to the global problem, is called globalization.

Two methods of globalization are discussed in this section:

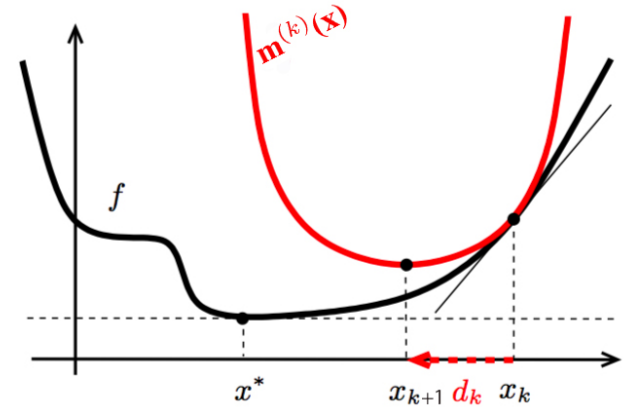

Fig. 1. Example of local approximation $\mathbf{m}^{(k)}(\mathbf{x})$ by Taylor-Young

the line search and the trust region approaches, a third method of globalization known as the proximal algorithms which is the main part of our contribution.

\section{A. Globalization by line search}

In the line search approach, a descent direction $\mathbf{d}^{(k)}$ is constructed, and a search is made along this direction starting from $\mathrm{x}^{(k)}$ to find a new iterate whose value of the objective function is smaller. The direction $\mathbf{d}^{(k)}$ is a direction of descent if there exists $\mathrm{C} \succ 0$ such that:

$$
\left.\left.f\left(x^{(k)}+\rho^{(k)} d^{(k)}\right)<f\left(x^{(k)}\right), \forall \rho \in\right] 0, C\right]
$$

Which simply becomes, in the case where $\mathrm{f}$ is differentiable in $\mathbf{x}^{(k)}$,

$$
d^{(k) T} \nabla_{x} f\left(x^{(k)}\right) \leq 0
$$

Many algorithms belonging to this category such as, The gradient method. This method consider a starting point $\mathbf{x}^{(0)}$, and try to minimize the cost function $f$ using a descent direction $\mathbf{d}^{(k)}=-g^{(k)}$ such as

$$
x^{(k+1)}=x^{(k)}-\rho^{(k)} g^{(k)},
$$

where $\rho^{(k)}$ is the descent step of the method and $\mathbf{g}^{(k)}$ is the gradient of the function $f$.

Although these methods are conceptually very simple and can be programmed directly, but they are often slow in practice [9]. Therefore, The Conjugate Gradient method (CG) is used instead. This second method of line search is very efficient for solving (1) especially when $\mathbf{N}$ is large and they must converge in at most $\mathbf{N}$ iterations, where $\mathbf{N}$ is the dimension of the unknown vector. The search direction has the following

$\mathbf{d}^{(k)}= \begin{cases}-g^{(k)} & \text { if } k=0 \\ -g^{(k)}+\beta^{(k)} d^{(k-1)} & \text { if } k \geq 1\end{cases}$

With the coefficient of conjugation:

$$
\beta^{(k)}=\frac{\left(g^{T}\right)^{(k)} g^{(k)}}{\left(g^{T}\right)^{(k-1)} g^{(k-1)}}
$$

The third method in this category is The Quasi-Newton method. It consist in imitating the Newton method [10], where the optimization of a function is obtained from the successive minimizations of its second-order approximation. Indeed, the disadvantage of the direction of Newton is that it requires 
knowledge of the Hessian of the objective function, the calculation of this matrix can be complicated and costly. Moreover, for some problems, the criterion is not twice differentiable. This motivated the emergence of the Quasi-Newton methods [9] which define the direction by:

$$
d^{(k)}=-\left(B^{-1}\right)^{k} g^{(k)}
$$

where $B$ is the approximation to the Hessian matrix. Readers seeking clarifications on the methods developed in this section are invited to consult the work of Stoer and Bulirsch [9] for example.

Formally the algorithm model based on line search is provided by algorithm 1

algorithm 1 : Choice of an initial iterate $x_{0} \in R^{n}$ and a small parameter $\epsilon>0$. Initialization $\mathrm{k}=1$.

- Step 1: Convergence test : if $\left\|\nabla f\left(x^{(k)}\right)\right\|_{2}<\epsilon$ stop of the algorithm

- $\quad$ Step 2: determine a descent direction $d_{k}$

- $\quad$ Step 3: determine a step of progression $\rho_{k}$ such that the function $f$ decreases sufficiently

- $\quad$ Step 4: determine a new iterated $x^{(k+1)}=x^{(k)}+$ $\rho^{(k)} d^{(k)}$

- $\quad$ Step 5: set $\mathrm{k}=\mathrm{k}+1$ and return to step 1 .

Among the different methods of searching for the approximate step length $\rho^{(k)}$. We use the backtracking method [12] which is very simple and effective. This method allows us to obtain a good approximation of the step $\rho^{(k)}$ at a lower cost. It depends on two constants $\alpha$ and $\beta$, with $0<\alpha<0.5$ and $0<\beta<1$.

This method is called backtracking, because it starts with a step $\rho^{(k)}$ large enough at the beginning, then this value is reduced by the factor $\beta$ such as $\rho^{(k)}=\rho^{(k)} \beta$, until the descent condition (4) is verified. A summary of the backtracking method is given in the following table:

\begin{tabular}{|l|}
\hline backtracking method \\
\hline 1. Given a descent direction $\mathbf{d}$ for $\mathrm{f}$, \\
$\alpha \in[0,0.5], \beta \in[0,1]$ \\
2. Initialize $: \rho=1$ \\
3. Do : \\
$\rho=\rho \beta$ \\
While $\mathrm{f}\left(\mathrm{x}^{(k)}+\rho \mathrm{d}\right)<\mathrm{f}\left(\mathrm{x}^{(k)}\right)+\alpha \rho g^{T} d$ \\
\hline
\end{tabular}

\section{B. Globalization by Trust Region}

Optimization methods by trust region are based on a simple idea. At each iteration, the local approximation $\mathbf{m}^{(k)}(\mathrm{x})$ (Figure 1 ) is considered as reliable in a given domain of determined validity. A trust region, for which the size is adapted according to the iterations, and with some assumptions, the global convergence of this approach to a local minimum can be rigorously established [12]. At each iteration, the algorithm defines a local approximation $\mathbf{m}^{(k)}(\mathrm{x})$ which has the aim to approach the objective function in a trust region

$$
B=\left\{x \in R^{n}:\left\|x-x^{k}\right\|_{k} \leq \Delta^{k}\right\}
$$

where $\Delta^{k}$ is the Confidence Region Radius (CRR) and where $\|\cdot\|_{k}$ is a norm depending on the iteration. A progression step $s^{(k)}$ is then computed by solving at each iteration the subproblem:

$$
\begin{array}{ll}
\underset{\mathbf{s}}{\operatorname{minimize}} & m^{(k)}\left(\mathbf{x}^{(\mathbf{k})}+\mathbf{s}\right) \\
\text { subject to } & \|s\|_{k} \leq \Delta^{(k)}
\end{array}
$$

Thereafter, the objective function $f\left(\tilde{x}^{(k)}\right)$ is computed at the test point

$$
\widetilde{x}^{(k)}=x^{(k)}+s^{(k)},
$$

and compared to the value predicted by the local approximation $\mathbf{m}^{(k)}(\mathrm{x})$. If a sufficient reduction of the objective function is obtained, the test point is accepted as the next iterate and the CRR increased or maintained constant. Otherwise, the test point is rejected and the CRR is contracted, in the hope that the local approximation will give better predictions over a smaller region [12].

Formally the algorithm model based on trust region is provided by algorithm 2

Algorithm 2 : Let a starting point $\mathbf{x}^{(0)}$, an initial CRR $\Delta^{(0)}$ and the constants $\eta_{1}, \eta_{2}, \gamma_{1}, \gamma_{2}$ which satisfy the conditions

$$
0<\eta_{1} \leq \eta_{2}<1 \quad \text { and } \quad 0<\gamma_{1} \leq \gamma_{2}<1
$$

Calculate $f\left(x^{(0)}\right)$ and initialize $\mathrm{k}=1$.

- Step 1: Define the local approximation. Choose the norm $\|\cdot\|_{k}$ and define a local approximation $\mathbf{m}^{(k)}$ in $B^{(k)}$.

- Step 2: Calculation of a progress step. Calculate a step $\mathrm{s}^{(k)}$ reducing sufficiently the local approximation $\mathrm{m}^{(k)}$ and such that: $\widetilde{x}^{(k)}=\mathrm{x}^{(k)}+\mathrm{s}^{(k)} \in \mathrm{B}^{(k)}$.

- Step 3: Accept or reject the test point. Evaluate $f\left(\tilde{x}^{(k)}\right)$ and define the ratio

$$
\rho^{(k)}=\frac{f\left(x^{(k)}\right)-f\left(\tilde{x}^{(k)}\right)}{m^{(k)}(x)^{(k)}-m^{(k)}\left(\tilde{x}^{(k)}\right)}
$$

If $\rho^{(k)} \geq \eta_{1}$, define $\mathrm{x}^{(k+1)}=\tilde{x}^{(k)}$; otherwise, $\mathrm{x}^{(k+1)}=\mathrm{x}^{(k)}$.

- Step 4: Update the confidence region radius. Choosing:

$$
\Delta^{(k+1)} \in \begin{cases}{\left[\Delta^{(k)},+\infty[\right.} & \text { if } \rho^{(k)} \geq \eta_{2} \\ {\left[\gamma_{2} \Delta^{(k)}, \Delta^{(k)}\right]} & \text { if } \rho^{(k)} \in\left[\eta_{1}, \eta_{2}\right] \\ {\left[\gamma_{1} \Delta^{(k)}, \gamma_{2} \Delta^{(k)}\right]} & \text { if } \rho^{(k)}<\eta_{1}\end{cases}
$$

- $\quad$ Step 5: set $\mathrm{k}=\mathrm{k}+1$ and return to step 1 .

We must also mention that there are other techniques of globalization alongside line search and trust region. Among these techniques, those called proximal point algorithms. Which are very close, in their minds to the methods of the trust region. The so-called proximal methods are already present in the Martinet thesis [13] but the algorithm of the proximal point finds its full foundations in the work of Rockafellar [14]. In its 


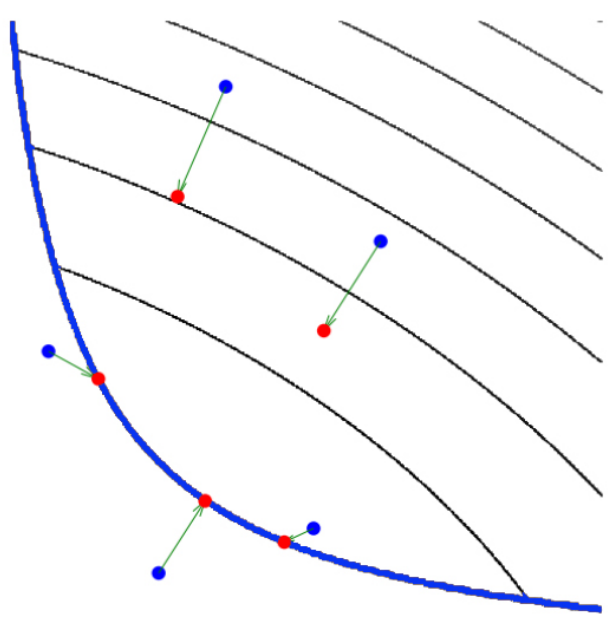

Fig. 2. Evaluating a proximal point at various points [15]

generality, this algorithm is developed to search for a zero of a monotonous maximal operator. Formally, let assume that $f$ be a closed proper convex function. The proximal point $\operatorname{prox}_{f}$ $: R^{N} \mapsto R^{N}$ of $\mathrm{f}$ is defined by

$$
\operatorname{prox}_{f}(v)=\arg \min _{x}\left(f(x)+\frac{1}{2 \mu}\|x-v\|_{2}^{2}\right),
$$

where $\|\cdot\|_{2}$ is the usual Euclidean norm. The function minimized on the righthand side is strongly convex and not everywhere infinite, so it has a unique minimizer for every $\mathrm{v} \in$ $R^{N}$ [15]. (Figure 2) represents what a proximal point does. The black lines are level curves of a convex function $\mathrm{f}$, the blue line indicates the boundary of its domain. Evaluating the proximal at the blue points moves them to the corresponding red points. The three points in the domain of the function stay in the domain and move towards the minimum of the function, while the other two move to the boundary of the domain and towards the minimum of the function. The parameter $\mu$ controls the stretch on which the proximal point maps points towards the minimum of $\mathrm{f}$. With larger values of $\mu$ associated with mapped points near the minimum, and smaller values giving a smaller movement towards the minimum.

\section{THE PROPOSED ALGORITHM}

The proposed algorithm is a variation of the proximal point algorithm by a suitable adaptation, where the variable $\mathbf{v}$ in the basic proximal [15] is replaced by the previous iterate and by controlling the progression of the algorithm by a new and flexible strategy. In this context, the algorithm is characterized by a basic iteration of the form

$$
\arg \min _{x} f(x)+\frac{1}{2 \mu^{(k)}}\left\|x-x^{(k)}\right\|^{2} .
$$

The distance term is introduced in order to regularize the function $f(x)$ and thus ensure the existence and uniqueness of the minimum $\mathbf{x}^{(k+1)}$ [16].

Adapting $\mu$ at each iteration (not fixing it) increases the results of convergence rates. For this purpose we analyzed the important role that $\mu$ plays in the control of the extent to which the proximal point maps points towards the minimum of $f$ (Figure 2), therefore we do suggest starting with a small $\mu$ (i.e. important penalization) then test if the next point is near to the minimum, if that is the case, we increase $\mu$ (weak penalization) otherwise we continue with the same $\mu$.

The proposed algorithm model based on proximal point is provided as follows:

Algorithm 3 : Let a starting point $\mathbf{x}^{(0)}$ and an $\epsilon$ fixed,

- Step 1: Initialization $\mathrm{k}=1$, the proximal algorithm generate a sequence $\left\{\mathbf{x}^{(k)}\right\}$ of iterations, by calculating $\mathbf{x}^{(k+1)}$ from $\mathbf{x}^{(k)}$ by:

$$
x^{(k+1)}=\arg \min _{x} f(x)+\frac{1}{2 \mu^{(k)}}\left\|x-x^{(k)}\right\|^{2}
$$

- $\quad$ Step 2:

$$
\begin{aligned}
& \text { if }\left\|\mathbf{x}^{(k+1)}-\mathbf{x}^{(k)}\right\|^{2} \leq \epsilon \text { then } \\
& \mu^{(k+1)}=2^{*} \mu^{(k)} \\
& \text { else } \\
& \mu^{(k+1)}=\mu^{(k)} \\
& \text { end if }
\end{aligned}
$$

- Step 3: set $\mathrm{k}=\mathrm{k}+1$ and return to step 1 .

In Step 2, we calculate the direction $\mathbf{d}$ by the conjugate gradient, and the step length $\rho$ by the backtracking method. We can see some similarity between the proximal methods and the trust region methods, the first penalize the distance between two iterates while the second confines the iterate $\mathbf{x}^{(k+1)}$ around $\mathbf{x}^{(k)}$ by a constraint. Where, on the one hand, the intensity of the penalty is governed by the parameter $\mu^{(k)}$ which adapts from iteration to iteration. Small values of $\mu^{(k)}$ provoke a strong penalty whose effects are similar to a trust region of small radius $\Delta^{(k)}$.

Regularization of proximal methods acts as a penalization -i.e. a form of constraint that is weak and easy to parameterizeWhereas trust region use a strong, impracticable and impassable constraint [15]. We can see that our proposed algorithm benefits from the advantages of the line search for the calculation of the direction and the step length and also from the advantages of the trust region by controlling the added regularization.

\section{REsults AND Discussion}

In this section, we present experimental results and several simulations for the various iterative methods for optimization. We illustrate the behavior of the different algorithms presented before, in order to highlight the impact of several factors on their performance, and we finish by introducing the simulations of our algorithm in comparison with the other relevant algorithms.

Simulation was done in Matlab on a computer with Intel i5 CPU $(2.6 \mathrm{GHz})$ and $10 \mathrm{~GB}$ memory running 64bit Mac OS. For simplicity, we have applied our algorithm to the quadratic functions because it is the basis for most of the nonlinear iterative algorithms which tries to find the solution by seeking for the solution of an approximation quadratic function in a local point. As long as the algorithm used to find the solution of the quadratic function is fast as long as it positively influences the resolution of the nonlinear functions 


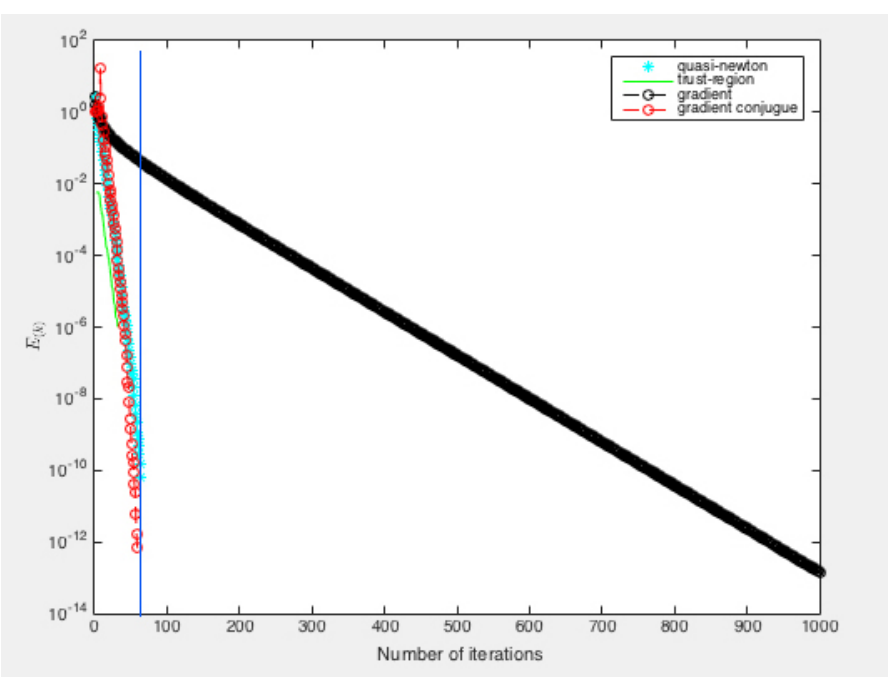

Fig. 3. Performance index according to the number of iterations

since the latter must find the solution of a quadratic function (approximation $\left.\mathbf{m}^{(k)}(\mathbf{x})\right)$ at each iteration.

To have the possibility of comparing the different algorithms, we need a performance index. For all scenarios. We choose to use an error index - by a prior knowledge of the global minimum $x^{*}$ of each tested function - defined as:

$$
E_{(k)}=\frac{\left\|x^{(k)}-x^{*}\right\|_{2}^{2}}{\left\|x^{*}\right\|_{2}}
$$

When the performance index $E_{(k)}$ is close to 0, the results obtained are said to be better. So we must give a threshold from which we consider that the global minimum of our function is reached, for example $\epsilon=10^{-6}$. As a result, the stop criterion will be $E_{(k)}<\epsilon$. Note also that the results are obtained after 20 Monte Carlo iterations.

For the first simulation, we compare the algorithms already present in the literature to have a validation of projection between the theoretical and experimental results, and also to conclude on the performance of each algorithms. In that case, we consider a matrix of size $100 \times 100$ of a quadratic function and we compare the convergence of the four algorithms with respect to a threshold of precise iterations. Figure 3 shows the convergence of these algorithms with respect to the threshold which is Threshold Iter $=70$ iterations. By comparing the four curves obtained in this figure, we note that for 70 iterations, the gradient algorithm tends to an error of about $10^{-1}$, the trust region algorithm tends to $10^{-6}$, the quasi-newton algorithm tends to $10^{-10}$ while the conjugate gradient algorithm reaches an error Of $10^{-12}$. These results allow us to conclude that the algorithm of the conjugate gradient is more efficient than the others.

In the second simulation we consider a matrix of size $60 \times 60$ and we compare the convergence of the three relevant algorithms of literature with our proposed algorithm, to situate our algorithm in relation to the algorithms already studied previously. Figure 4 shows the convergence of these algorithms. By comparing the four curves obtained in this figure, we observe that our proposed algorithm with the progress

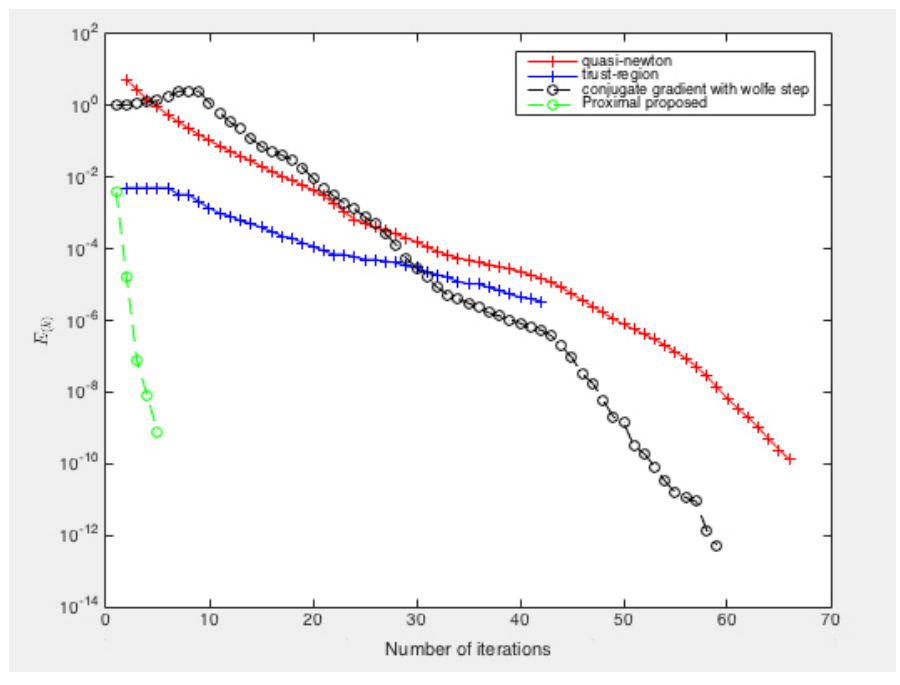

Fig. 4. Performance index according to the number of iterations

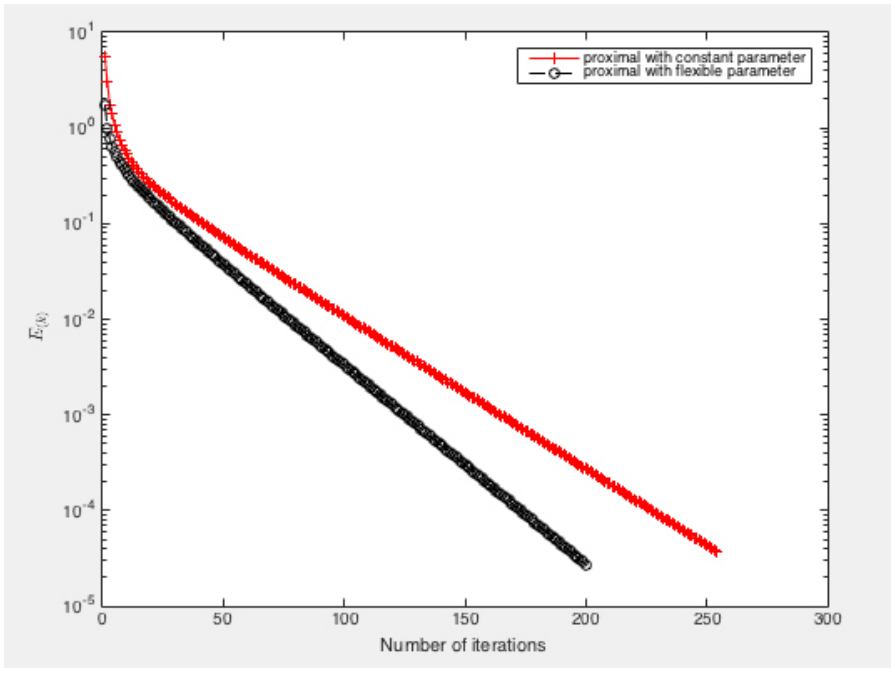

Fig. 5. Simulation of the proximal algorithm with both constant and flexible progression of $\mu^{(k)}$

strategy requires a minimum of iterations with respect to the other algorithms. All these results allow us to conclude that our algorithm is more efficient than the others.

For the final simulation, we consider a matrix of size $60 \times 80$ and we compare the convergence of the two proposed algorithms, the results of the simulations show us that the proximal with our strategy of advancing the parameter $\mu^{(k)}$ gives accurate results even better than with the proximal with a constant $\mu^{(k)}$, and we can also observe that the proximal with our strategy needs fewer iterations 200 iterations against 250 for the the proximal with a constant $\mu^{(k)}$.

To make a good comparison between the optimization algorithms studied, we evaluated the computation time of the second simulation, and the results of this evaluation is presented in the following table 


\begin{tabular}{|l|c|}
\hline Algorithm & Calculation time (s) \\
\hline Conjugate Gradient (CG) & 18.27 \\
Quasi-Newton & 17.48 \\
Trust Region & 15.32 \\
Proposed Algorithm & 16.38 \\
\hline
\end{tabular}

by analyzing the results of the simulations and the computation time of all the algorithms, we can well remark that the proposed algorithm is a good candidate for ensuring both accuracy and the rapid convergence to solve the unconstrained optimization problem.

\section{CONCLUSION}

In this paper, we presented an algorithm of proximal with a simple and effective progression strategy. This algorithm has the advantage of being fast and accurate compared to other iterative optimization algorithms.

The main advantage of using the proximal algorithm with the progression strategy is in its simplicity to implement and also in its improved results. In addition, we have made a complete comparison of the simulations results of the first order iterative algorithms presented in the literature with those of our algorithm, which proved the accuracy and speed of convergence. Furthermore, the simulation results between the proximal algorithm with a constant progression step and with the proposed strategy for advancing this parameter showed an additional improvement in the accuracy of the results.

In conclusion, the proximal algorithm seems to be a competitive candidate for optimization algorithms. We expect that the proximal algorithm can be extended to other more complex functions and also to other applications.

\section{REFERENCES}

[1] G. K. Smyth, "Optimization and nonlinear equations," Wiley StatsRef: Statistics Reference Online, pp. 1-9, 2015.

[2] R. K. Joseph Sloan, David Kesler and A. Rahimi, "A numerical optimization-based methodology for application robustification: Transforming applications for error tolerance," in IEEE/IFIP International Conference on Dependable Systems and Networks, August 2010.

[3] S. B. Stefania Sardellitti, Gesualdo Scutari, "Joint optimization of radio and computational resources for multicell mobile-edge computing," IEEE Trans. on Signal and Information Processing over Networks, Dec 2014.

[4] R. B. Leila Ismail, "Implementation and performance evaluation of a distributed conjugate gradient method in a cloud computing environment," Software: Practice and Experience, vol. 43, pp. 281-304, March 2013.

[5] A. G. S Cui and A. Bahai, "Energy-constrained modulation optimization," IEEE Transactions on Wireless Communications, vol. 4, Sept 2005.

[6] R. Z. CK Ho, "Optimal energy allocation for wireless communications with energy harvesting constraints," IEEE Transactions on Signal Processing, 2012.

[7] Y. X. et al., "Cognitive internet of things: A new paradigm beyond connection," IEEE Internet of Things Journal, vol. 1, April 2014.

[8] B. Conway, "A survey of methods available for the numerical optimization of continuous dynamic systems," Journal of Optimization Theory and Applications, 2012.

[9] J. Nocedal and S. J. Wright, Numerical Optimization. Springer Series in Operations Research. Springer, 1999.

[10] A. Ahookhosh, M., "An efficient nonmonotone trust-region method for unconstrained optimization," Numerical Algorithms, pp. 523-540, 2012.

[11] J. Stoer and R. Bulirsch, Introduction to numerical analysis. SpringerVerlag, New York, third dition, 2002.
[12] G. e. a. Conn, A. R., Trust-Region Methods. MPS/SIAM Series on Optimization. SIAM, Philadelphia, USA, 2000.

[13] B. Martinet, Algorithms for solving optimization and minimax problems. $\mathrm{PhD}$ thesis, University of Grenoble, 1972.

[14] R. T. Rockafellar, "Monotone operators and the proximal point algorithm," SIAM Journal on Control and Optimization, pp. 877-898, 1976.

[15] N. Parikh and S. Boyd, "Proximal algorithms," Found. Trends Optim, vol. 1, 2013.

[16] S. Boyd and L. Vandenberghe., "Convex optimization," Cambridge University Press, 2004. 